\title{
Aortic Arch Calcification Predicts the Renal Function Progression in Patients with Stage 3 to 5 Chronic Kidney Disease
}

\author{
Lung-Chih Li, ${ }^{1}$ Yueh-Ting Lee, ${ }^{1}$ Yi-Wei Lee, ${ }^{2,3}$ Chia-An Chou, ${ }^{1}$ and Chien-Te Lee \\ ${ }^{1}$ Division of Nephrology, Department of Internal Medicine, Kaohsiung Chang Gung Memorial Hospital and \\ Chang Gung University College of Medicine, 123 Ta-Pei Road, Niao-Sung District, Kaohsiung 833, Taiwan \\ ${ }^{2}$ Department of Biomedical Imaging and Radiological Sciences, National Yang Ming University, Taipei 102, Taiwan \\ ${ }^{3}$ Department of Radiology, Kaohsiung Chang Gung Memorial Hospital and Chang Gung University College of Medicine, \\ Kaohsiung 833, Taiwan \\ Correspondence should be addressed to Chien-Te Lee; chientel@gmail.com
}

Received 30 July 2014; Accepted 19 November 2014

Academic Editor: Wolfgang Neuhofer

Copyright (C) 2015 Lung-Chih Li et al. This is an open access article distributed under the Creative Commons Attribution License, which permits unrestricted use, distribution, and reproduction in any medium, provided the original work is properly cited.

\begin{abstract}
Introduction. The presence of aortic arch calcification (AoAC) and cardiomegaly on chest radiography has been demonstrated as important risk factors for cardiovascular mortality in patients with chronic kidney disease (CKD). However, the interrelationship among AoAC, cardiomegaly, and renal function progression remains unclear. The aim of this study is to assess whether AoAC and cardiomegaly are independently associated with the renal function progression in patients with stages 3-5 CKD. Methods. We retrospectively determined AoAC and cardiomegaly by chest X-ray in 237 patients, followed up for at least three years without entering dialysis and classified into 4 groups according to the presence or absence of AoAC and cardiomegaly. The change in renal function was measured by the slope of estimated glomerular filtration rate (eGFR). Results. Of the 237 patients, the rate of eGFR decline was significantly higher in the group with coexistence of AoAC and cardiomegaly than any other groups. Baseline AoAC and proteinuria were independently associated with eGFR decline. AoAC were independently determined by age, eGFR slope, and cardiomegaly. Conclusions. The coexistence of AoAC and cardiomegaly is associated with faster eGFR decline. AoAC is an independent determinant of renal outcomes in patients with CKD stages 3-5.
\end{abstract}

\section{Introduction}

Cardiovascular disease is the major cause of mortality in patients with all stages of chronic kidney disease (CKD) [1]. Several well-known traditional risk factors [2], such as old age, hypertension, diabetes, cigarette smoking, and dyslipidemia, have been found to partially, yet not fully, explain the high prevalence of cardiovascular events in these patients. Recently, the roles of nontraditional risk factors have been more emphasized [3]. Of these factors, vascular calcification and cardiomegaly have emerged as new potential risk factors for predicting the cardiovascular events in CKD patients $[4,5]$.

Vascular calcification is highly prevalent in patients with CKD compared to general population. More than half of CKD patients even before the start of dialysis and up to $80-90 \%$ of ESRD patients have various degrees of vascular calcification [6, 7]. Recently, aortic arch calcification (AoAC) on chest X-ray, which is an inexpensive and noninvasive exam, has been found to reflect the magnitude of whole aortic calcification in general population and CKD patients [8]. We and others have shown that AoAC is independently associated with cardiovascular and all-cause mortality in both CKD and ESRD patients [9-11]. However, the relationship of presence of AoAC and renal function progression remains unclear.

Increased cardiothoracic ratio (CTR) $(>50 \%)$, another easily available parameter on chest radiography, is a marker of cardiomegaly and is considered predictive of left ventricular systolic dysfunction in chronic heart failure patients [12]. High CTR independently correlates with increased left ventricular mass and target organ damage in hypertensive patients [13]. Recently, increased CTR is shown to be correlated with malnutrition and inflammation and is 
predictive of two-year all-cause mortality in ESRD patients with hemodialysis and peritoneal dialysis $[5,14]$. However, whether the CTR can predict the renal function deterioration in CKD patients remains unknown.

The aim of this study is to evaluate whether the presence of AoAC and/or cardiomegaly on chest radiographs can predict the renal function progression in patients with stages 3-5 CKD. We hypothesized that there is a possible interrelationship between AoAC and/or cardiomegaly on chest radiographs and progression of renal function so that it may be a useful tool for anticipation of renal outcome and for early intervention in these patients.

\section{Subjects and Methods}

2.1. Ethics Statement. This study was carried out in accordance with the Declaration of Helsinki and approved by the Institution Review Board of Kaohsiung Chang-Gung Memorial Hospital (IRB102-3070B).

2.2. Study Patients and Design. All CKD patients over 18 years of age were reviewed from the outpatients of Nephrology Department of Kaohsiung Chang-Gung Memorial Hospital between September 2006 and December 2012 in this retrospective observational study. We screened 598 predialysis patients with stage 3 to $5 \mathrm{CKD}$ according to the National Kidney Foundation-Kidney Disease Outcomes Quality (KDOQI) guidelines [15]. Among them, a total of 269 patients who have been followed up for at least 3 years without entering end stage kidney disease or mortality were included in this study. We classified our patients with evidence of kidney injury lasting for more than 3 months into CKD stage 3 , stage 4 , and stage 5 , based on estimated glomerular filtration rate (eGFR) level of 30 to 59,15 to 29 , and $<15$, respectively. Eighteen patients with less than three eGFR measurements during the follow-up period were excluded. Four patients who lack chest X-ray and 10 patients who had acute renal failure, defined by increase of creatinine up to 2 times or decrease of eGFR up to $50 \%$ in one month, were also excluded. Finally, 237 patients were enrolled in this study. Later the study patients were stratified into 4 groups according to whether they have AoAC with the absence or presence of cardiomegaly on chest $\mathrm{X}$-rays.

\subsection{Demographic and Clinical Data Collection. Demographic} and medical data including age, gender, and comorbid conditions were obtained from medical records or interviews with patients. Body mass index (BMI) was calculated as weight/height ${ }^{2}\left(\mathrm{~kg} / \mathrm{m}^{2}\right)$. Blood was drawn after over 8 hours overnight fasting and the following laboratory data were measured from blood samples: hemoglobin, creatinine, blood urea nitrogen, calcium, phosphorus, sodium, potassium, albumin, total cholesterol, triglyceride, low density lipoprotein (LDL) cholesterol, and high density lipoprotein (HDL) cholesterol. Mean values of two measurements within the three months before or after chest radiography were used for analysis. The value of eGFR was calculated based on the simplified equation in the Modification of Diet in Renal Disease (MDRD) study [16], which is eGFR $\mathrm{mL} / \mathrm{min} / 1.73 \mathrm{~m}^{2}=$ $175 \times$ serum creatinine $e^{-1.154} \times$ age $^{-0.203} \times 0.742$ (if female). Urine total protein to creatinine ratio (UPCR) was calculated as urine total protein (mg)/urine creatinine (g). The blood and urine sample were obtained on the same day.

\subsection{Evaluation of AoAC and Cardiomegaly by Chest $X$ -} Ray. One experienced radiologist and one trained medical doctor blinded to the patients' clinical data independently reviewed the posterior-anterior (P-A) chest plain films of those CKD patients taken at the beginning of entering CKD care program. Calcification of aortic arch was assessed using a scale developed by Ogawa et al. [17]. The scale, dividing aortic arch into 16 sections by circumference, was attached to the aortic arch on chest X-rays and the number of sectors with calcification was counted. A calcification score of 2 and above was regarded as presence of calcification while a calcification score of 0 and 1 was regarded as absence of calcification. Cardiomegaly was defined as a transverse diameter of the cardiac silhouette greater than or equal to $50 \%$ of the transverse diameter of the chest on the P-A chest radiograph. To examine the intrareader variability, randomly selected 50 chest X-rays were examined by both readers. Five $\mathrm{X}$-rays were scored differently on calcification score with a maximum difference of score \pm 2 ; two X-rays were judged differently on increased CTR due to borderline cardiomegaly. The discrepancies between the two observers were resolved by a third independent reader.

2.5. Statistical Analysis. Statistics analysis was performed using SPSS for windows version 18.0 (SPSS Inc., Chicago, IL, USA). Continuous variables were expressed as mean \pm $\mathrm{SD}$, and categorical variables were expressed as a number (percentage). Multiple comparisons among the four study groups were conducted by one-way analysis of variance (ANOVA) followed by post hoc test adjusted with Bonferroni's correction. Linear regression analysis was used to identify the factors associated with decline in kidney function and logistic regression analysis was used to determine the factors related to aortic calcification. Significant variables in univariate analysis were selected for multivariate analysis. A difference was considered significant if the $P$ value was less than 0.05 .

\section{Results}

The comparison of the baseline characteristics among the four study groups is demonstrated in Table 1. A total of 237 patients with stage 3 to $5 \mathrm{CKD}$ were recruited. The mean age was $67.6 \pm 13.2$ years. The average age was oldest in the group with coexistence of AoAC and cardiomegaly (72.6 \pm 11.6 years old), followed by AoAC only (71.1 \pm 10.0 years old), cardiomegaly only $(60.8 \pm 13.7$ years old), and none of each (60.2 \pm 13.2 years old). Patients with coexistence of AoAC and cardiomegaly had the highest BMI $(26.5 \pm$ $5.0 \mathrm{~kg} / \mathrm{m}^{2}$ ), the highest proportion of diabetes $(58 \%)$, and the highest UPCR $(1908.4 \pm 2714.6 \mathrm{mg} / \mathrm{g})$ among the four 
TABLE 1: Clinical features and biochemical data of patients among study groups.

\begin{tabular}{|c|c|c|c|c|c|c|}
\hline & $\begin{array}{c}\text { Total } \\
(N=237)\end{array}$ & $\begin{array}{c}\operatorname{AoAC}(-) \mathrm{CM}(-) \\
(N=65)\end{array}$ & $\begin{array}{c}\operatorname{AoAC}(-) \mathrm{CM}(+) \\
(N=26)\end{array}$ & $\begin{array}{c}\operatorname{AoAC}(+) \mathrm{CM}(-) \\
(N=55)\end{array}$ & $\begin{array}{c}\operatorname{AoAC}(+) \mathrm{CM}(+) \\
(N=91)\end{array}$ & $P$ value \\
\hline Age (years) & $67.6 \pm 13.2$ & $60.2 \pm 13.2$ & $60.8 \pm 13.7$ & $71.1 \pm 10.0^{\mathrm{ab}}$ & $72.6 \pm 11.6^{\mathrm{ab}}$ & $<0.0001$ \\
\hline Gender, male (\%) & $148(62)$ & $46(71)$ & $15(58)$ & $36(66)$ & $51(56)$ & 0.264 \\
\hline BMI $\left(\mathrm{kg} / \mathrm{m}^{2}\right)$ & $24.5 \pm 4.3$ & $23.7 \pm 3.5$ & $24.9 \pm 3.8$ & $23.4 \pm 3.2$ & $26.5 \pm 5.0^{\mathrm{ac}}$ & $<0.0001$ \\
\hline Diabetes (\%) & $109(46)$ & $24(37)$ & $10(39)$ & $22(40)$ & $53(58)$ & 0.010 \\
\hline UPCR (mg/g) & $1313.3 \pm 2197.8$ & $846.1 \pm 1319.6$ & $1600.2 \pm 3129.3$ & $887.9 \pm 1387.2$ & $1908.4 \pm 2714.6^{\mathrm{a}}$ & 0.028 \\
\hline Hemoglobin (mg/dL) & $11.8 \pm 2.4$ & $12.2 \pm 2.2$ & $12.2 \pm 2.8$ & $11.6 \pm 2.5$ & $11.4 \pm 2.4$ & 0.149 \\
\hline Serum creatinine $(\mathrm{mg} / \mathrm{dL})$ & $2.5 \pm 1.2$ & $2.3 \pm 1.2$ & $2.7 \pm 1.6$ & $2.7 \pm 1.3$ & $2.5 \pm 1.1$ & 0.207 \\
\hline $\mathrm{eGFR}\left(\mathrm{mL} / \mathrm{min} / 1.73 \mathrm{~m}^{2}\right)$ & $30.3 \pm 15.6$ & $34.6 \pm 15.6$ & $31.1 \pm 19.7$ & $27.9 \pm 14.9$ & $29.3 \pm 14.4$ & 0.086 \\
\hline Sodium $(\mathrm{meq} / \mathrm{L})$ & $140.3 \pm 2.8$ & $140.8 \pm 2.1$ & $140.5 \pm 2.6$ & $140.3 \pm 2.6$ & $140.0 \pm 3.2$ & 0.397 \\
\hline Potassium (meq/L) & $4.4 \pm 0.6$ & $4.4 \pm 0.6$ & $4.3 \pm 0.6$ & $4.4 \pm 0.5$ & $4.5 \pm 0.6$ & 0.805 \\
\hline Calcium (mg/dL) & $8.9 \pm 0.5$ & $8.9 \pm 0.5$ & $9.0 \pm 0.5$ & $8.8 \pm 0.4$ & $8.9 \pm 0.6$ & 0.584 \\
\hline Phosphorus (mg/dL) & $3.7 \pm 0.8$ & $3.6 \pm 0.9$ & $3.7 \pm 0.9$ & $3.7 \pm 0.7$ & $3.7 \pm 0.8$ & 0.897 \\
\hline $\mathrm{Ca} \times \mathrm{P}$ & $32.2 \pm 8.4$ & $32.2 \pm 8.7$ & $32.7 \pm 8.0$ & $32.6 \pm 5.8$ & $31.7 \pm 9.6$ & 0.935 \\
\hline Albumin $(\mathrm{mg} / \mathrm{dL})$ & $4.1 \pm 0.5$ & $4.2 \pm 0.5$ & $4.1 \pm 0.5$ & $4.2 \pm 0.4$ & $3.9 \pm 0.6^{\mathrm{ac}}$ & 0.004 \\
\hline Total cholesterol (mg/dL) & $194.8 \pm 61.7$ & $209.9 \pm 89.2$ & $181.4 \pm 30.4$ & $190.7 \pm 45.2$ & $190.6 \pm 52.5$ & 0.186 \\
\hline Triglyceride (mg/dL) & $163.4 \pm 118.5$ & $164.1 \pm 118.2$ & $190.6 \pm 121.9$ & $150.8 \pm 132.7$ & $163.8 \pm 108.7$ & 0.637 \\
\hline $\mathrm{HDL}$ & $48.2 \pm 15.3$ & $49.7 \pm 16.4$ & $46.8 \pm 15.1$ & $51.9 \pm 14.7$ & $45.1 \pm 14.4$ & 0.124 \\
\hline LDL & $115.8 \pm 53.9$ & $134.7 \pm 81.2$ & $95.7 \pm 26.7^{\mathrm{a}}$ & $114.2 \pm 34.8$ & $108.7 \pm 39.9$ & 0.023 \\
\hline
\end{tabular}

BMI: body mass index; UPCR: urine protein-creatinine ratio; eGFR: estimated glomerular filtration rate; HDL: high density lipoprotein cholesterol; LDL: low density lipoprotein cholesterol; AoAC: aortic arch calcification; CM: cardiomegaly; ${ }^{\mathrm{a}} P<0.05$ compared with $\operatorname{AoAC}(-) \mathrm{CM}(-)$; ${ }^{\mathrm{b}} P<0.05$ compared with $\operatorname{AoAC}(-) \mathrm{CM}(+) ;{ }^{\mathrm{c}} \mathrm{P}<0.05$ compared with $\mathrm{AoAC}(+) \mathrm{CM}(-)$.

groups. Albumin was the lowest in patients with coexistence of AoAC and cardiomegaly $(3.9 \pm 0.6 \mathrm{mg} / \mathrm{dL})$, while it was the highest in patients without AoAC or cardiomegaly ( $4.2 \pm$ $0.5 \mathrm{mg} / \mathrm{dL}$ ). LDL cholesterol was the highest among patients without AoAC or cardiomegaly $(134.7 \pm 81.2 \mathrm{mg} / \mathrm{dL})$ whereas patients with cardiomegaly only had the lowest levels of LDL $(95.7 \pm 26.7)$. There was no significant difference in gender, hemoglobin, creatinine, eGFR, sodium, potassium, calcium, phosphorus, total cholesterol, triglyceride, or HDL among four groups.

3.1. Risks of Renal Function Decline. Figure 1 illustrates the eGFR slope in (a) total, (b) stage 3, and (c) stages 4 and 5 CKD patients. In all patients, the eGFR slopes in 4 groups were $-0.8 \pm 2.2,-1.6 \pm 2.4,-2.2 \pm 2.4$, and $-3.8 \pm$ $2.7 \mathrm{~mL} / \mathrm{min} / 1.73 \mathrm{~m}^{2}$, respectively. The slope of eGFR decline was significantly higher in patients with coexistence of AoAC and cardiomegaly than all other groups. Patients with AoAC only had faster eGFR deterioration than patients without AoAC or cardiomegaly.

In stage $3 \mathrm{CKD}$ patients, the eGFR slopes in 4 groups were $-0.5 \pm 2.3,-0.5 \pm 3.2,-2.1 \pm 3.4$, and $-4.8 \pm 3.1 \mathrm{~mL} / \mathrm{min} / 1.73 \mathrm{~m}^{2}$, respectively. The group with coexistence of AoAC and cardiomegaly had a significantly greater eGFR decline than all other groups. However, in patients with stages 4 and 5 CKD, patients with coexistence of AoAC and cardiomegaly had only significantly greater eGFR decline than patients without AoAC or cardiomegaly $(-3.1 \pm 2.2$ versus $-1.0 \pm$ $\left.2.0 \mathrm{~mL} / \mathrm{min} / 1.73 \mathrm{~m}^{2}, P<0.01\right)$.
Table 2 shows the determinants of eGFR slope in all patients. Greater renal function progression had larger negative slope. In the univariate analysis, the eGFR slope had a significant positive correlation with albumin and negative correlation with age, diabetes, BMI, UPCR, baseline eGFR, AoAC, and cardiomegaly. In the multivariate analysis, the eGFR slope was correlated independently with UPCR $(\beta=$ $-0.203, P=0.012)$ and $\operatorname{AoAC}(\beta=-0.224, P=0.007)$, indicating that patients with more proteinuria and higher AoAC had faster progression of renal function.

3.2. Determinants of Aortic Arch Calcification. The determinants of AoAC are demonstrated in Table 3. The univariate regression analysis showed that old age, high BMI, presence of diabetes, cardiomegaly, and lower eGFR slope were associated with AoAC. In the multivariate study, AoAC was independently correlated with old age (odds ratio: 1.080, $P<$ 0.0001 ), cardiomegaly (odds ratio: $2.491, P=0.01$ ), and a lower eGFR slope (odds ratio: $0.813, P<0.0001$ ).

\section{Discussion}

In the present study for a cohort of participants with stages 3$5 \mathrm{CKD}$, we found that both AoAC and cardiomegaly on chest $\mathrm{X}$-ray are associated with renal function progression. AoAC is independently associated with renal function decline after adjustments for various covariates, suggesting that AoAC is not only a consequence of CKD but itself is predictive of renal function progression. 


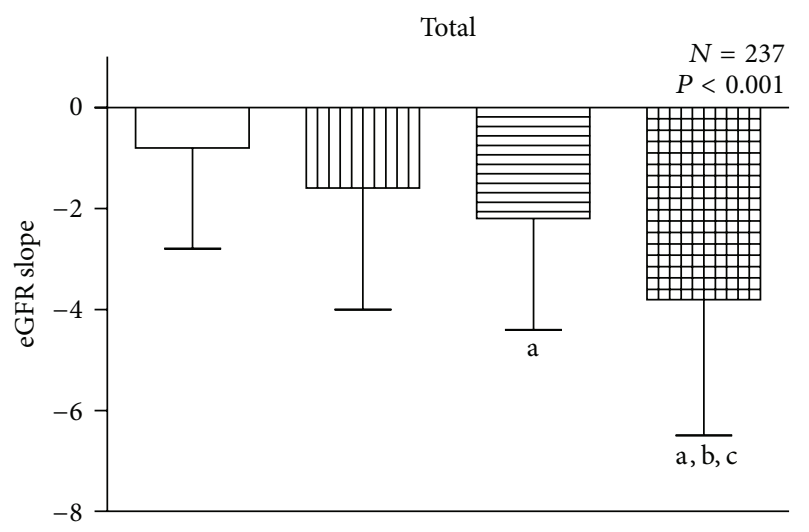

(a)

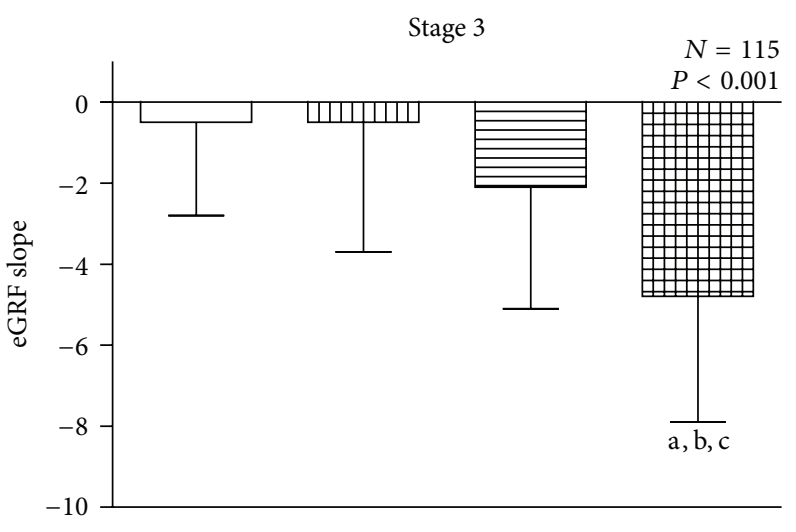

(b)

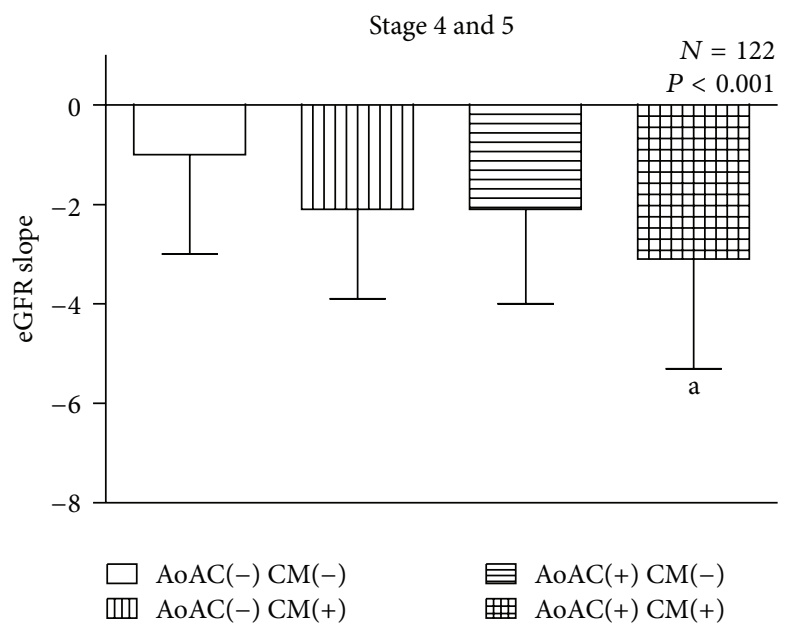

(c)

FIGURE 1: The eGFR slopes among 4 study groups: (a) in total 237 patients; (b) in patients with stage 3 CKD; (c) in patients with stages 4 and 5 CKD. ${ }^{a} P<0.05$ compared with the group without AoAC nor CM. ${ }^{b} P<0.05$ compared with the group with $\mathrm{CM}$ only. ${ }^{\mathrm{c}} \mathrm{P}<0.05$ compared with the group with AoAC only. eGFR, estimated glomerular filtration; AoAC, aortic arch calcification; CM, cardiomegaly.

Multiple factors are identified and involved during the progression of CKD. There are modifiable factors such as hypertension and hyperglycemia. Adequate intervention can retard the decline of renal function and reduce cardiovascular mortality. It has been well recognized that detailed evaluation and appropriate management of risk factors are effective strategies for the treatment of CKD.

Calcification of aortic arch is not uncommon in general elderly population. It has been reported that $20-30 \%$ of people older than 65 years have calcification in the aorta [8]. In CKD patients, the prevalence of AoAC is even higher. In our cohort, more than half $(60 \%)$ of the patients had various degrees of AoAC and, in agreement with previous studies [18], patients with AoAC are older than patients without AoAC. However, after adjusting the factor of age, AoAC still independently predicts the renal function deterioration in our study cohort.

AoAC may represent part of the vascular calcification in the whole body. A number of studies have shown an interrelationship between vascular calcification and increased arterial stiffness. Arterial stiffness, resulting from vascular calcification, is a powerful predictor of poor cardiovascular outcome [7]. Increased pulse pressure and pulse wave velocity, two indicators of arterial stiffness, were reported to be risk factors of rapid decline of renal function in CKD patients $[19,20]$ and in patients with relative normal baseline renal function $[21,22]$. Although the mechanisms by which arterial stiffness causes renal damage remains incompletely understood, it has been proposed that the microcirculation, in which glomerular capillaries are positioned between afferent and efferent arterioles, increases susceptibility to injury under long-term increased pulse pressure [23]. These may explain, at least in part, that AoAC is associated with rapid renal function decline.

Of note, patients with coexistence of AoAC and increased CTR (cardiomegaly) are associated with greater renal function decline compared with patients with either AoAC or cardiomegaly or none of each. The eGFR decline of patients with coexistence of AoAC and cardiomegaly in stage 3, but not in later stages (stages 4 and 5), is close to rapid 
TABLE 2: Determinants associated with eGFR slope in study patients (linear regression).

\begin{tabular}{|c|c|c|c|c|}
\hline & \multicolumn{2}{|c|}{ Univariate } & \multicolumn{2}{|c|}{ Multivariate } \\
\hline & Standardized coefficient $\beta$ & $P$ value & Standardized coefficient $\beta$ & $P$ value \\
\hline Age (per 1-year increase) & -0.152 & 0.015 & -0.103 & 0.201 \\
\hline Male gender & 0.034 & 0.593 & - & - \\
\hline Diabetes & -0.235 & $<0.0001$ & -0.102 & 0.176 \\
\hline BMI (per $1 \mathrm{~m}^{2}$ increase) & -0.194 & 0.003 & -0.118 & 0.124 \\
\hline $\mathrm{UPCR}(\mathrm{mg} / \mathrm{g})$ & -5.729 & $<0.0001$ & -0.203 & 0.012 \\
\hline Baseline eGFR & -0.010 & 0.037 & -0.049 & 0.518 \\
\hline Calcium & -0.051 & 0.446 & - & - \\
\hline Phosphorus & 0.039 & 0.564 & - & - \\
\hline $\mathrm{Ca} \times \mathrm{P}$ & 0.009 & 0.890 & - & - \\
\hline Albumin & 0.133 & 0.047 & 0.100 & 0.211 \\
\hline Total cholesterol (mg/dL) & 0.093 & 0.175 & - & - \\
\hline Triglyceride (mg/dL) & -0.070 & 0.306 & - & - \\
\hline $\mathrm{HDL}$ & -0.003 & 0.969 & - & - \\
\hline LDL & 0.145 & 0.051 & - & - \\
\hline AoAC & -0.253 & $<0.0001$ & -0.224 & 0.007 \\
\hline Cardiomegaly & -0.131 & $<0.0001$ & -0.039 & 0.620 \\
\hline
\end{tabular}

BMI: body mass index; UPCR: urine protein-creatinine ratio; eGFR: estimated glomerular filtration rate; HDL: high density lipoprotein cholesterol; LDL: low density lipoprotein cholesterol, AoAC: aortic arch calcification.

TABLE 3: Determinants of aortic arch calcification in study patients (logistic regression).

\begin{tabular}{|c|c|c|c|c|}
\hline & \multicolumn{2}{|c|}{ Univariate } & \multicolumn{2}{|c|}{ Multivariate } \\
\hline & Odds ratio (95\% CI) & $P$ value & Odds ratio (95\% CI) & $P$ value \\
\hline Age (per 1-year increase) & $1.085(1.058-1.114)$ & $<0.0001$ & $1.080(1.048-1.111)$ & $<0.0001$ \\
\hline Male gender & $0.737(0.438-1.241)$ & 0.252 & - & - \\
\hline Diabetes & $1.533(1.045-2.904)$ & 0.001 & $0.821(0.426-1.595)$ & 0.575 \\
\hline $\mathrm{BMI}$ (per $1 \mathrm{~m}^{2}$ increase) & $1.071(1.002-1.145)$ & 0.042 & $0.993(0.923-1.085)$ & 0.875 \\
\hline UPCR (mg/g) & $1.319(0.741-2.346)$ & 0.347 & - & - \\
\hline Baseline eGFR & $0.989(0.973-1.004)$ & 0.144 & - & - \\
\hline CKD stage & $1.196(0.868-1.650)$ & 0.274 & - & - \\
\hline eGFR slope & $0.842(0.777-0.911)$ & $<0.0001$ & $0.813(0.773-0.938)$ & $<0.0001$ \\
\hline Calcium & $1.036(0.648-1.657)$ & 0.882 & - & - \\
\hline Phosphorus & $0.910(0.676-1.226)$ & 0.536 & - & - \\
\hline $\mathrm{Ca} \times \mathrm{P}$ & $0.982(0.951-1.013)$ & 0.251 & - & - \\
\hline Albumin & $0.770(0.464-1.276)$ & 0.310 & - & - \\
\hline Total cholesterol (mg/dL) & $0.997(0.993-1.002)$ & 0.273 & - & - \\
\hline Triglyceride (mg/dL) & $0.999(0.997-1.002)$ & 0.567 & - & - \\
\hline $\mathrm{HDL}$ & $0.999(0.980-1.019)$ & 0.952 & - & - \\
\hline LDL & $0.996(0.989-1.002)$ & 0.166 & - & - \\
\hline Cardiomegaly & $3.911(2.289-6.683)$ & $<0.0001$ & $2.491(1.326-4.996)$ & 0.010 \\
\hline
\end{tabular}

BMI: body mass index; UPCR: urine protein-creatinine ratio; CKD: chronic kidney disease; eGFR: estimated glomerular filtration rate; HDL: high density lipoprotein cholesterol; LDL: low density lipoprotein cholesterol.

progression of renal function, defined by a decline in eGFR of more than $5 \mathrm{~mL} / \mathrm{min} / 1.73 \mathrm{~m}^{2}$ per year by K-DIQO guideline [24], implicating that presence of AoAC and cardiomegaly in the early stage of CKD may be associated with worse renal outcome. Although increased CTR is not an independent determinant of rapid renal function decline, AoAC is correlated with cardiomegaly in the current study. It has been shown that aortic arch calcification volume measured by multidirector computed tomography is independently correlated with left ventricular diastolic dysfunction [25]. Vascular calcification was also proved to cause hemodynamic alternations such as reduced compliance of large arteries and automatic dysfunction in hemodialysis patients [26]. Moreover, pulse wave velocity, as a determinant of arterial stiffness, 
is highly associated with LV hypertrophy in hemodialysis patients $[25,27]$. Cardiomegaly may develop with AoAC and correlate with renal function progression.

Proteinuria is another independent determinant of rapid renal function deterioration in the present study. Baseline proteinuria is known to be associated with the risk of CKD progression in both nondiabetic [28] and diabetic $[29,30]$ patients. Recently Chen et al. also reported that various degrees of proteinuria may have different impact on cardiovascular events and all-cause mortality [31]. However, the interrelationship between proteinuria and vascular calcification remains unclear. One recent study has shown that coronary artery calcification is associated with albuminuria in type 2 diabetic patients [32]; however, our study did not support the role of proteinuria in AoAC. Instead, age, eGFR slope, and increased CTR are the independent determinants of AoAC.

Several limitations need to be acknowledged. First, since patients with early entering dialysis ( $<3$ years) were excluded, the effects of AoAC on renal function may be underestimated in patients with late stage CKD (e.g., stage 5). Second, the use of AoAC and increased CTR on chest X-ray are not as sensitive and specific as other modalities such as CT scanning for calcification and cardiac sonography for cardiomegaly. Third, it is a relatively small-sized study, which may not have strong power to show the significance in some way. In addition, due to its observational and retrospective design, it may be subject to potential confounding from missing variables. Nevertheless we were still able to demonstrate an independent relation between AoAC and renal function progression by using this noninvasive and nonexpansive modality.

\section{Conclusion}

This study presents for the first time that baseline AoAC measured on chest radiography and proteinuria are independently associated with faster eGFR decline in patient with stages 3-5 CKD. AoAC are determined by age, eGFR slope, and cardiomegaly but not by proteinuria. The evaluation of AoAC on chest radiography may represent a simple, cheap, and noninvasive method to predict the renal function progression in these patients.

\section{Conflict of Interests}

The authors declare that there is no conflict of interests regarding the publication of this paper.

\section{References}

[1] R. Vanholder, Z. Massy, A. Argiles et al., "Chronic kidney disease as cause of cardiovascular morbidity and mortality," Nephrology Dialysis Transplantation, vol. 20, no. 6, pp. 10481056, 2005.

[2] P. Jungers, T. N. Khoa, D. Joly, G. Choukroun, V. Witko-Sarsat, and Z. A. Massy, "Atherosclerotic complications in chronic renal failure: epidemiology and predictive factors," Advances in Nephrology from the Necker Hospital, vol. 30, pp. 177-199, 2000.
[3] P. Stenvinkel, J. J. Carrero, J. Axelsson, B. Lindholm, O. Heimbürger, and Z. Massy, "Emerging biomarkers for evaluating cardiovascular risk in the chronic kidney disease patient: how do new pieces fit into the uremic puzzle?" Clinical Journal of the American Society of Nephrology, vol. 3, no. 2, pp. 505-521, 2008.

[4] G. M. London, J. Blacher, B. Pannier, A. P. Guérin, S. J. Marchais, and M. E. Safar, "Arterial wave reflections and survival in endstage renal failure," Hypertension, vol. 38, no. 3, pp. 434-438, 2001.

[5] K.-H. Chen, D.-T. Lin-Tan, W.-H. Huang et al., "Cardiothoracic ratio, malnutrition, inflammation, and two-year mortality in non-diabetic patients on maintenance hemodialysis," Kidney and Blood Pressure Research, vol. 31, no. 3, pp. 143-151, 2008.

[6] J. S. Garland, R. M. Holden, P. A. Groome et al., "Prevalence and associations of coronary artery calcification in patients with stages 3 to 5 CKD without cardiovascular disease," American Journal of Kidney Diseases, vol. 52, no. 5, pp. 849-858, 2008.

[7] J. Blacher, A. P. Guerin, B. Pannier, S. J. Marchais, and G. M. London, "Arterial calcifications, arterial stiffness, and cardiovascular risk in end-stage renal disease," Hypertension, vol. 38, no. 4, pp. 938-942, 2001.

[8] C. Iribarren, S. Sidney, B. Sternfeld, and W. S. Browner, "Calcification of the aortic arch: risk factors and association with coronary heart disease, stroke, and peripheral vascular disease," Journal of the American Medical Association, vol. 283, no. 21, pp. 2810-2815, 2000.

[9] C.-T. Lee, C.-C. Huang, C.-Y. Hsu et al., "Calcification of the aortic arch predicts cardiovascular and all-cause mortality in chronic hemodialysis patients," Cardiorenal Medicine, vol. 4, no. 1, pp. 34-42, 2014.

[10] M. J. Lee, D. H. Shin, S. J. Kim et al., "Progression of aortic arch calcification over 1 year is an independent predictor of mortality in incident peritoneal dialysis patients," PLoS ONE, vol. 7, no. 11, Article ID e48793, 2012.

[11] M. K. Sigrist, M. W. Taal, P. Bungay, and C. W. McIntyre, "Progressive vascular calcification over 2 years is associated with arterial stiffening and increased mortality in patients with stages 4 and 5 chronic kidney disease," Clinical Journal of the American Society of Nephrology, vol. 2, no. 6, pp. 1241-1248, 2007.

[12] S. Shah, M. K. Davies, D. Cartwright, and P. Nightingale, "Management of chronic heart failure in the community: role of a hospital based open access heart failure service," Heart, vol. 90, no. 7, pp. 755-759, 2004.

[13] B. L. Rayner, H. Goodman, and L. H. Opie, "The chest radiograph: a useful investigation in the evaluation of hypertensive patients," The American Journal of Hypertension, vol. 17, no. 6, pp. 507-510, 2004.

[14] K. H. Chen, C. C. Hung, D. T. Lin-Tan et al., "Cardiothoracic ratio association with mortality in patients on maintenance peritoneal dialysis," Therapeutic Apheresis and Dialysis, vol. 15, no. 1, pp. 81-88, 2011.

[15] A. S. Levey, L. A. Stevens, and J. Coresh, "Conceptual Model of CKD: applications and implications," The American Journal of Kidney Diseases, vol. 53, no. 3, pp. S4-S16, 2009.

[16] A. S. Levey, J. P. Bosch, J. B. Lewis, T. Greene, N. Rogers, and D. Roth, "A more accurate method to estimate glomerular filtration rate from serum creatinine: a new prediction equation," Annals of Internal Medicine, vol. 130, no. 6, pp. 461-470, 1999.

[17] T. Ogawa, H. Ishida, N. Matsuda et al., "Simple evaluation of aortic arch calcification by chest radiography in hemodialysis 
patients," Hemodialysis International, vol. 13, no. 3, pp. 301-306, 2009.

[18] K. Iijima, H. Hashimoto, M. Hashimoto et al., "Aortic arch calcification detectable on chest X-ray is a strong independent predictor of cardiovascular events beyond traditional risk factors," Atherosclerosis, vol. 210, no. 1, pp. 137-144, 2010.

[19] N. Arulkumaran, R. Diwakar, Z. Tahir, M. Mohamed, J. C. Kaski, and D. Banerjee, "Pulse pressure and progression of chronic kidney disease," Journal of Nephrology, vol. 23, no. 2, pp. 189-193, 2010.

[20] M. L. Ford, L. A. Tomlinson, T. P. E. Chapman, C. Rajkumar, and S. G. Holt, "Aortic stiffness is independently associated with rate of renal function decline in chronic kidney disease stages 3 and 4," Hypertension, vol. 55, no. 5, pp. 1110-1115, 2010.

[21] M. Madero, C. Peralta, R. Katz et al., "Association of arterial rigidity with incident kidney disease and kidney function decline: the health ABC study," Clinical Journal of the American Society of Nephrology, vol. 8, no. 3, pp. 424-433, 2013.

[22] H. Tomiyama, H. Tanaka, H. Hashimoto et al., "Arterial stiffness and declines in individuals with normal renal function/early chronic kidney disease," Atherosclerosis, vol. 212, no. 1, pp. 345350, 2010.

[23] M. F. O’Rourke and M. E. Safar, "Relationship between aortic stiffening and microvascular disease in brain and kidney: cause and logic of therapy," Hypertension, vol. 46, no. 1, pp. 200-204, 2005.

[24] "Chapter 2: definition, identification, and prediction of CKD progression," Kidney International Supplements, vol. 3, no. 1, pp. 63-72, 2013.

[25] A. Fujiu, T. Ogawa, N. Matsuda, Y. Ando, and K. Nitta, "Aortic arch calcification and arterial stiffness are independent factors for diastolic left ventricular dysfunction in chronic hemodialysis patients," Circulation Journal, vol. 72, no. 11, pp. 1768-1772, 2008

[26] L. J. Chesterton, M. K. Sigrist, T. Bennett, M. W. Taal, and C. W. McIntyre, "Reduced baroreflex sensitivity is associated with increased vascular calcification and arterial stiffness," Nephrology Dialysis Transplantation, vol. 20, no. 6, pp. 1140-1147, 2005.

[27] P. Raggi, A. Bellasi, E. Ferramosca, T. Islam, P. Muntner, and G. A. Block, "Association of pulse wave velocity with vascular and valvular calcification in hemodialysis patients," Kidney International, vol. 71, no. 8, pp. 802-807, 2007.

[28] P. Ruggenenti, A. Perna, L. Mosconi et al., "Proteinuria predicts end-stage renal failure in non-diabetic chronic nephropathies. The "gruppo Italiano di studi epidemiologici in nefrologia' (GISEN)," Kidney international, vol. 63, pp. S54-57, 1997.

[29] W. F. Keane, Z. Zhang, P. A. Lyle et al., "Risk scores for predicting outcomes in patients with type 2 diabetes and nephropathy: the RENAAL study," Clinical Journal of the American Society of Nephrology, vol. 1, no. 4, pp. 761-767, 2006.

[30] R. C. Atkins, E. M. Briganti, J. B. Lewis et al., "Proteinuria reduction and progression to renal failure in patients with type 2 diabetes mellitus and overt nephropathy," American Journal of Kidney Diseases, vol. 45, no. 2, pp. 281-287, 2005.

[31] S.-C. Chen, C.-C. Hung, Y.-C. Tsai et al., "Association of cholesterol levels with mortality and cardiovascular events among patients with CKD and different amounts of proteinuria," Clinical Journal of the American Society of Nephrology, vol. 8, no. 11, pp. 1915-1926, 2013.
[32] A. Dayan, B. Narin, M. Biteker, S. Aksoy, H. Fotbolcu, and D. Duman, "Coronary calcium score, albuminuria and inflammatory markers in type 2 diabetic patients: associations and prognostic implications," Diabetes Research and Clinical Practice, vol. 98, no. 1, pp. 98-103, 2012. 


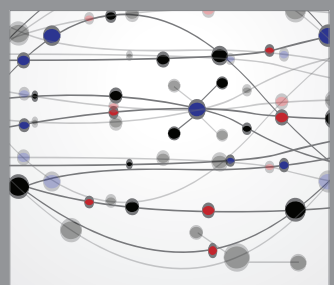

The Scientific World Journal
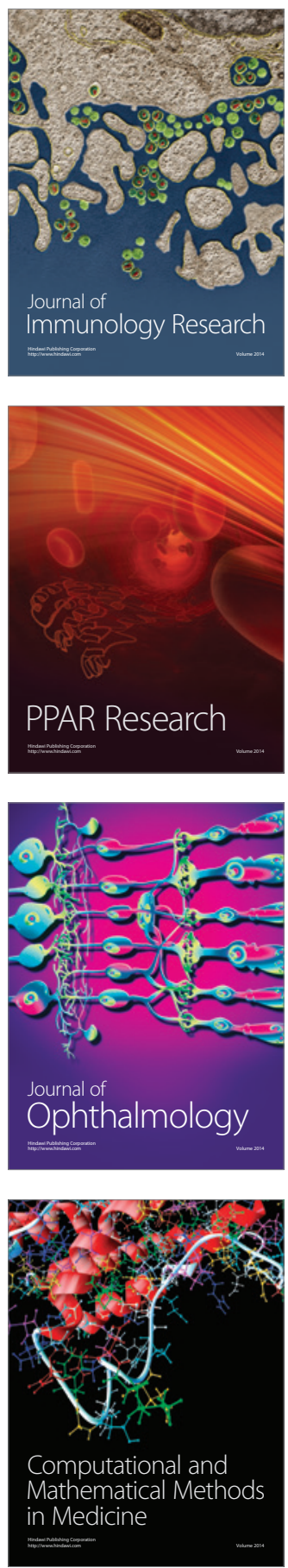

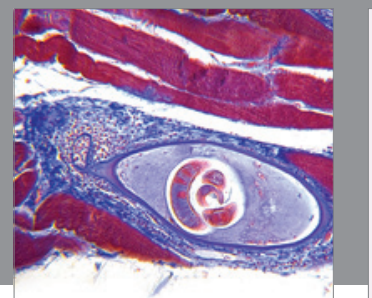

Gastroenterology

Research and Practice
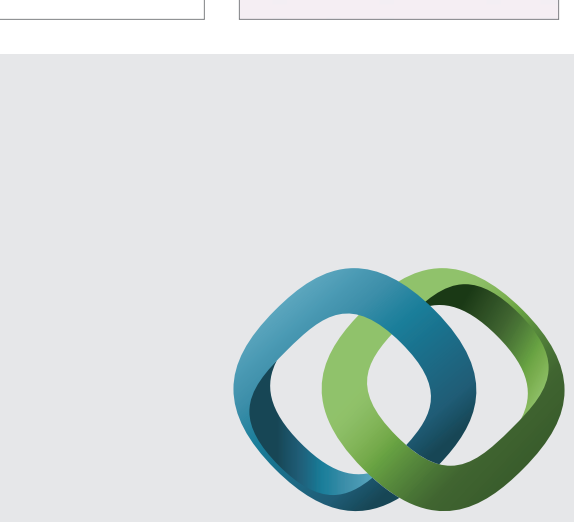

\section{Hindawi}

Submit your manuscripts at

http://www.hindawi.com
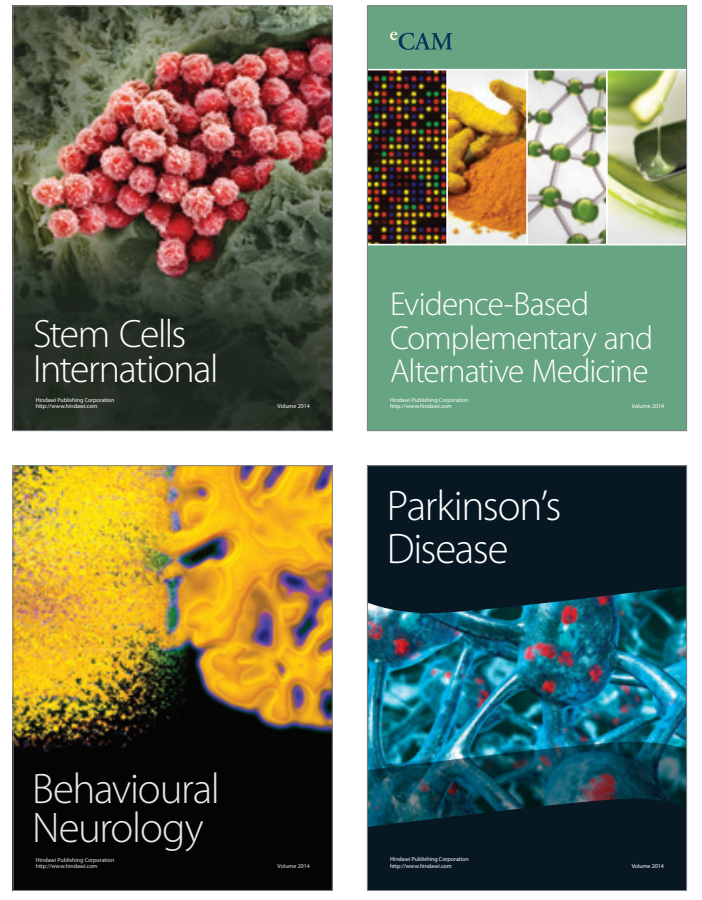
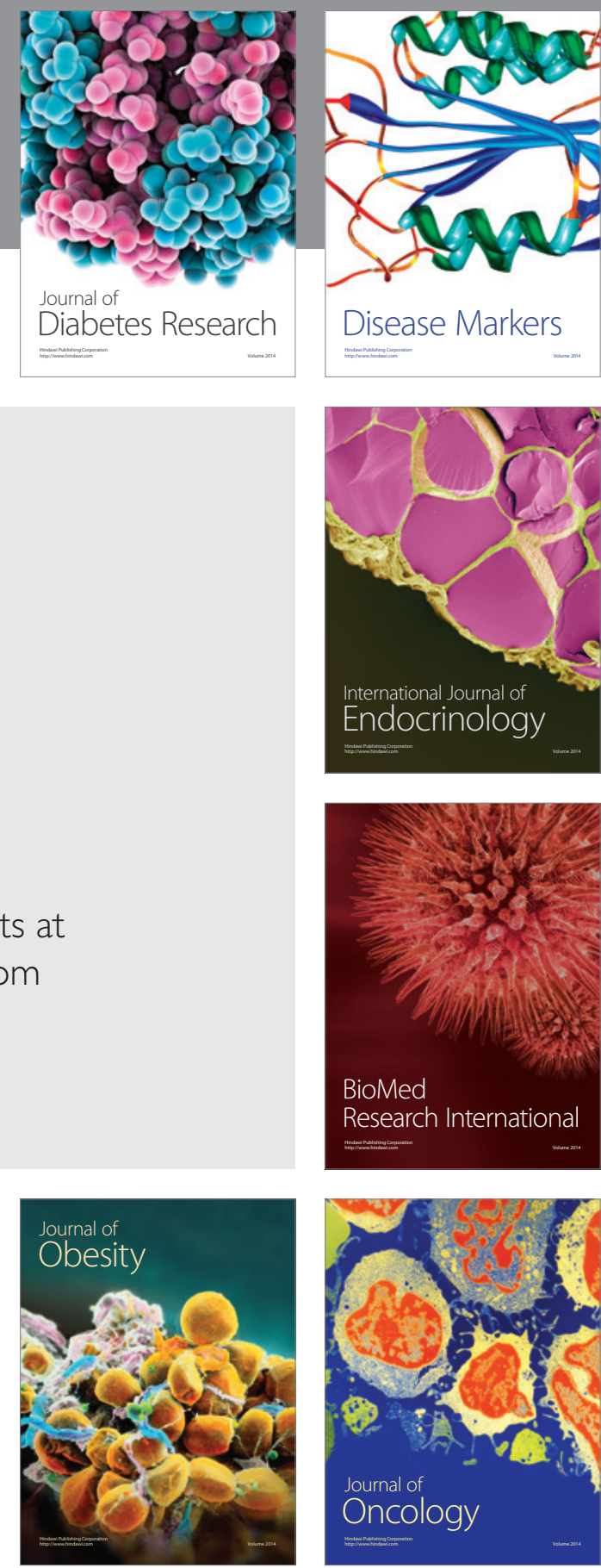

Disease Markers
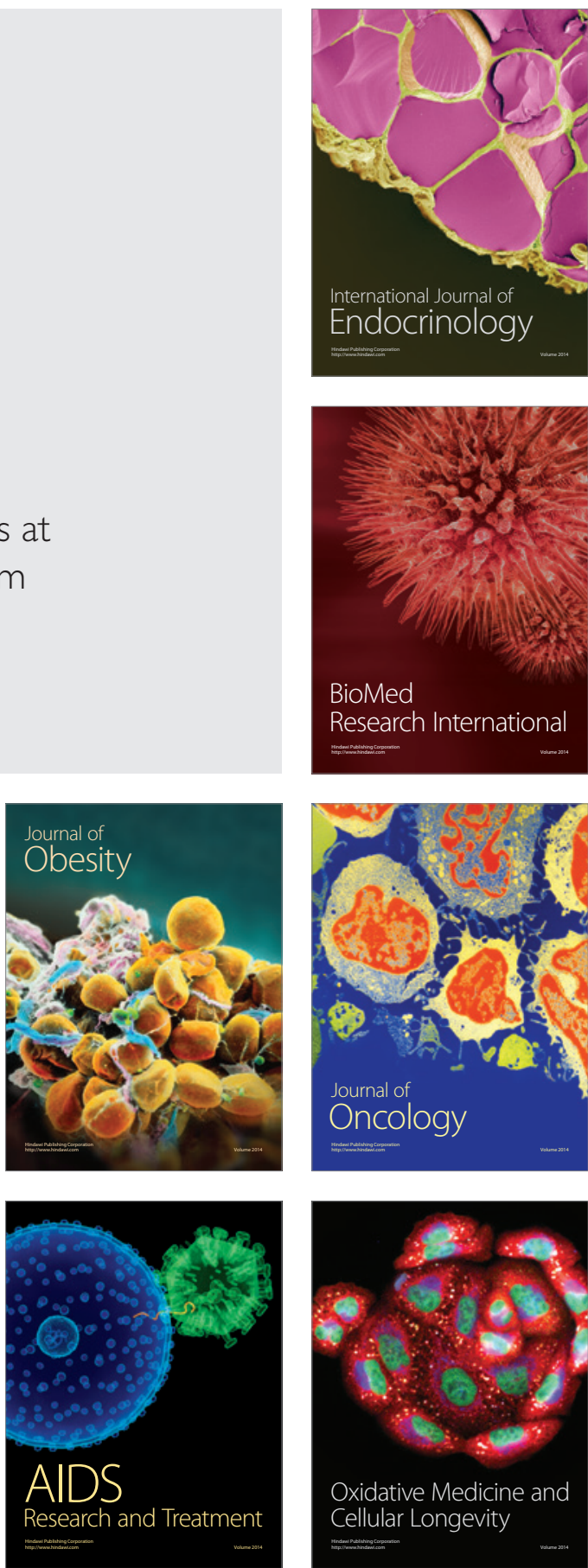